Reprint : from R.L. Amoroso , G. Hunter, M. Kafatos \& J-P Vigier (eds.), Gravitation and Cosmology: From the Hubble Radius to the Planck Scale, 2002 Dordrecht: Kluwer.

\title{
CAN ONE UNIFY GRAVITY AND ELECTROMAGNETIC FIELDS?
}

\author{
J-P. VIGIER \\ Université Paris VI - CNRS \\ Gravitation et Cosmologie Relativistes \\ Tour 22-12 4 ème étage - Boîte 142 \\ 4, place Jussieu, 75252 Paris Cedex 05 \\ R.L. AMOROSO \\ Noetic Advanced Studies Institute - Physics Lab \\ 120 Village Square MS 49, \\ Orinda, CA 94563-2502 USA \\ noeticj@mindspring.com
}

\begin{abstract}
This paper presents an attempt to unify gravity and electromagnetism associated with «holes » and «bumps » in the covariant density distribution of a real average covariant Dirac aether built with extended random elements filling flat spacetime. Some possible experimental tests are also discussed.
\end{abstract}

\section{Introduction}

The problem of the unification of gravity and electromagnetism into a single theory is as old as Modern Science itself and it has not been solved until now. Despite the initial discovery of similar forms of the Newton and Coulomb potential the two theories are still developping independently.

Until the present, unification has been attempted mainly (as a consequence of Einstein's discoveries) by Einstein himself [1], following Schrödinger [2], Maxwell [3] (and their present successors) within a frame associating electromagnetism with new geometrical properties of spacetime. The aim of the present paper is different. Following MacGrégor [4], Puthoff [5], and others, both fields are represented by fourvector field densities $A_{\mu}$; and one considers both types of phenomena as different types of motions within the same real physical zero-point field in flat spacetime, i.e. as two different « aether » types of collective perturbations carried by a single « aether » field moving in such a space. Since this approach suggests new types of experiments and yields an interpretation of unexplained new effects it will (perhaps), if confirmed, help to disantangle the present theoretical discussion.

This model has the following experimental basis :

I) The first basis (observational) is that the observable universe apparently does not change with distance [15] (as it should with big-bang type theories) and the ratio 
of the local $2.7^{\circ}$ microwave radiation is only isotropic in a specific absolute inertial frame $I_{0}$ : so that the velocity of light not only changes with its direction (which suggests a non-zero photon mass $m_{\gamma} \neq 0$ ) but is also isotropic in $I_{0}$, in time.

II) The second basis is that our essential instrument of (distant) observation (i.e. electromagnetic waves) is more complex than its initial discoverers (Maxwell and Ampère) thought. Newtons initial guess that light was both waves and particles (photons) was later confirmed by Einstein in 1905. The discovery by Fresnel that these waves were essentially transverse (i.e. with possible zero mass and invariant velocity of propagation) was later completed by de Broglie's and Einstein's discovery that one could write $E=h v=\mathrm{mc}^{2}$ (with $\mathrm{m}=\mathrm{m}_{0}\left(1-v^{2} / c^{2}\right)^{-1 / 2}$ ) so that individual massive photon's can be considered as piloted by real non zero-mass Maxwellian waves i.e. by new properties of the Sagnac effects in a recent experiment of Levit et al. [7] which shows that the electromagnetic field should be represented by a vector density $A_{\mu}$. As shown by Aharonov-Bohm effect, this implies that the electromagnetic field is not completely represented by the $\mu \nu$ fields $[6,7]$.

III) The third basis has its theoretical origin in the introduction by Dirac et al. of a real covariant chaotic physical « aether » which fills space-time, carries real physical observable wave-like and particle like (soliton-like) perturbations or local extended elements, whose four momenta and angular momenta are statistically and evently distributed on specific hyperbolic surfaces, at each given point, in all given inertial frames. This «vacuum» distribution thus appears, FAPP, as invariant isotropic chaotic and undetectable (except in specific physical cases) for all inertial observers. The form taken by an aether within Relativity Theory carries both particles and waves is now discribed in terms of collective motions on the top of a real essentially stochastic covariant background. Such an «aether» theoretically justifies the statistical productions of Quantum Mechanics (in its causal stochastic interpretation) and SED theory, and has a direct experimental justification in the Casimir effect. This implies a background friction (associated with absolute local conservation of total momentum and angular momentum) and collective motions which provide a new interpretation of the observed cosmological red-shift $[22,23]$ and yields new possibilities to interpret (also in terms of local frictions) the anomalous red-shifts observed by Arp, Tifft and other astronomers [8].

On these bases, we shall, in section 3, recall results showing that one can describe the gravitational results of General Relativity in Maxwellian terms. In section 4 we develop a possible unification model of both theories. Section 5 then contains a brief discussion of possible consequences of the preceding attempt. This aether is locally defined by a particular real Poincaré frame $I_{\mathrm{O}}$, in which (measured with real physical instruments) the velocity of light is identical in all directions at all observable frequencies. All observers tied to other frames passing through local inertial motions will see (measure) different space-time properties (associated with their velocity and 
orientations) defined by the corresponding Poincaré transformations. ${ }^{1}$ The local variations of physical properties of the aether correspond to local transitions relating differential inertial frames at neighbouring points.

\section{A Real Physical Aether In Flat Spacetime}

Since the starting point of this model is the existence of a real physical vacuum (or zero point field) built with extended wave-like individual elements $[9,10]$ centered on points in an external flat space-time, such elements can overlap and interact (i.e.carry) collective motions corresponding to excess (electromagnetic 'bumps') or defects (gravitational 'holes') in the average density of the local aether elements. The model can be described F.A.P.P. as a gas of extended elements within flat space-time. These elements can interact locally (i.e. carry collective motions) and the gas' local scalar density thus carries waves (and solitons) associated with excess (electromagnetic) or defects (gravitational) in density, with respect to the average local vacuum density. One thus defines field variables associated with these two possible (excess or defect) local density variations. The vector fields, for example, in this paper, represent localized excess or density defects w.r.t. the local vacuum density. This model thus implies:

a) a description of real physical vacuum properties in terms of real extended vacuum elements average behaviour.

b) a description of the behaviour of its collective defects (below average) associated with observed gravitational effects

c) a description of the behaviour of its collective excess (above average) associated with recently observed electromagnetic effects.

The introduction of such new concepts into Maxwell's equations and the description of gravitational fields along the same lines (in terms of vector fields $A_{\mu}$ ) suggests (as

we shall now see) a new type of unification of both theories. We shall discuss some of its prospects keeping in mind the restriction that, since new experiments are under way, it cannot yet be given a complete form. Instead of looking for a common geometrization of gravity and light (i.e. their unification within a unique form of extended space-time geometry) one could assume following Newton and Lorentz :

A) That the evolution of extended (fields) and of localized (sources) in terms of 1) vacuum (aether) 2) gravitational fields, 3) the electromagnetic field, reflects the time evolution (motions) and interactions of perturbations of a real material substance moving in a 3-dimensional flat space. This means that all three field and particle subelements are localized at given points, at each instant, in this 3 -space and move continuously (i.e. locally transform) according to causal laws ${ }^{2}$

\footnotetext{
1 To quote Kholmetsky « In order to pass from one arbitrary inertial frame $I_{1}$ to another one $I_{2}$ it is necessary to carry out the transformation from $I_{1}$ to the absolute frames $I_{O}$ and then from $I_{0}$ to $I_{2}$.

2 As a consequence of the failure of the geometrical unification program Einstein himself was still obliged in 1954 to consider the electromagnetic field as filling curved space-time. He never reached a final satisfying model.
} 
This assumption (distinction of space and fields) is now supported by the existence of a special particular experimental inertial cosmological frame $I_{0}$ in which - $\quad$ the $2.7^{\circ} \mathrm{K}$ microwave radiation frame is isotropic and non rotating - The average distribution of different types of galaxies (spiral, elliptical, Q.S.O's) is isotropic and does not change with distance [15].

- The observable anisotropy of the velocity of light propagation in different directions and around massive objects reflects the real motions of real fields described w.r.t. the $I_{0}$ frame in any real inertial Poincaré frame by covariant (local) four-vector scalar chaotic average density $\rho\left(x_{\mu}\right)$ around each absolute space-time point $x_{\mu}$ in $I_{0}$ i.e. by average four-vectors $A_{\mu}^{0}\left(x_{\alpha}\right)$ where ${ }^{0}$ denotes average measures taken in $I_{0} \cdot 3$

B) That all real physical observations rest on :

1. The utilisation of real physical apparatus based on electromagnetic fields and gravitational material with charged (or uncharged) particles.

2. On observers also built with the same material i.e. influenced by the said fields and particles.

In other terms all observers (and their observations, inertial or not) are an integral part of fields and particles since they are part of the same overall real field and particle distribution. This fact determines their relation with all real phenomena. A physical theory should explicitly provide (within its context) a definition of the means whereby the quantities with which the theory is built and can be measured. The properties of light rays and massive particles are thus sufficient to provide the means of making basic measurements. Since real clocks and rods are the real instruments utilized in physics, we shall thus first define, for an individual inertial observer, the behaviour of such instruments with respect to each other: since this determines, for every inertial observer possessing them, the behaviour, with respect to $I_{0}$,of the material fields around him.

As a consequence of the covariant distribution character observed in $I_{0}$, the very small resistance to motion and assumed non-zero photon rest mass, real spin of possible extended vacuum sub-elements and their internal possible motions (and associated local interactions) one can describe the four-momenta and angular momenta of all extended subelements passing through a small four-volume with a constant average density on a hyperboloid $\Sigma_{0}$. The four-momenta and angular momenta of extended elements are distributed at each point $P\left(x_{\mu}\right)$ with constant density $\rho\left(x_{\mu}\right)$ on space-like hyperboloids.

C) Following an idea of Noether the local analysis of moving fields and extended particles at each point by real observers tied to this point, is defined by local clocks

3 This implies 1) the existence of a basic high density of sub-elements in vacuum, 2) the existence of small density variations above (for light) and below (for gravity) the average density with the possibility to propagate densityvariation on the top of such a vacuum model as initially suggested by Dirac. 
and rods which move with the corresponding element. It is thus locally performed at each point of coordinates $x_{\mu}(\tau)$ which follows a world-line L.. To this point are attached local (in $I_{0}$ ) « internal » variables $b^{(\lambda)}$, which describe its neighbourhoods physical properties and thus depend on $\tau$. The evolution is given by $x_{\mu}\left(\dot{x}_{\mu}\right)$, $b^{\lambda}\left(\dot{b}^{\lambda}\right)$, where denotes the proper time dertivative w.r.t. $\tau$ when $x_{\mu}$ describes a world-line L.. A scalar Lag-rangian thus represents the evolution of the real physical medium in $I_{0}$, which depends on a local Lagrangian $L$ and is thus given by Poisson brackets. This description on $I_{0}$ is assumed to correspond to local space-time translations and four dimensional rotations which are determined by a Lagrangian $L$ invariant under the local group of Poincaré transformations (i.e. the inhomogeneous Lorentz group). They contain [15] :

1) the operators $P_{\mu}$ of infinitesimal translations of $X_{\mu} \quad$ only $\quad$ and $\quad$ can be described by $P_{\mu} \cdot X_{\lambda}=g_{\mu \lambda}$.

2) The operators $M_{\mu \nu}$ of infinitesimal four rotations in $I_{0}$ which act simultaneously on $X_{\mu}$ and on the internal variables. We have at $X_{\mu}$ :

$$
M_{\mu \nu} x_{\lambda}=x_{\mu} g_{v \lambda}-x_{\nu} g_{\mu \lambda}
$$

Their action on internal local variables depends on their choice.

3) A choice of $L$ leads to the momenta

$$
G_{\mu}=\frac{\partial L}{\partial \dot{x}_{\mu}} \quad \text { and } \quad \beta^{(\lambda)}=\frac{\partial L}{\partial \dot{b}^{(\lambda)}}
$$

yielding a constant impulsion vector

$$
G_{\lambda} P_{\mu} x_{\lambda}=G_{\lambda} g_{\mu \lambda}=G_{\mu}:
$$

and the total angular momentum:

$$
M_{\mu \nu}=G_{\lambda} M_{\mu v} x_{\lambda}+\beta^{(\lambda)} M_{\mu \nu} b^{(\lambda)},
$$

so that

$$
M_{\mu v}=x_{\mu} G_{v}-x_{v} G_{\mu}+S_{\mu v},
$$

with

$$
S_{\mu \nu}=\beta^{(\lambda)} M_{\mu v} \beta^{(\lambda)}
$$

These quantities satisfy the Inhomogeneous Lorentz group commutation relations 


$$
\begin{gathered}
{\left[P_{\mu}, P_{\lambda}\right]=0} \\
{\left[M_{\mu v}, P_{\alpha}\right]=g_{\alpha \beta} P_{v}-g_{\alpha \nu} P_{\mu}}
\end{gathered}
$$

i.e. Poisson Group Relations :

$$
\begin{gathered}
{\left[G_{\mu}, G_{\nu}\right]=0} \\
{\left[M_{\mu \nu}, G_{\alpha}\right]=g_{\alpha \beta} G_{\nu}-g_{\alpha \nu} G_{\mu}} \\
{\left[M_{\mu \nu}, M_{\alpha \beta}\right]=g_{\mu \alpha} M_{\nu \beta}+g_{\nu \beta} M_{\mu \alpha}-g_{\mu \beta} M_{\nu \alpha}-g_{\nu \alpha} M_{\mu \beta} .}
\end{gathered}
$$

With these quantities one can also define local conservation laws for « free » elements i.e.

$$
\begin{aligned}
& \dot{G}_{\mu}=0 \\
& \dot{M}_{\mu \nu}=0 \\
& \dot{S}_{\mu \nu}=G_{\mu} \dot{x}_{\nu}-G_{\mu} \dot{x}_{\mu} .
\end{aligned}
$$

and introduce a constant local mass term $\mathrm{M}_{0}$ with $G_{\mu} G_{\mu}=-M_{0}^{2} \cdot c^{2}$.

4) An associated center of gravity $y_{\mu}$ is defined by the introduction of the four-vector

$$
R_{\mu}=\left(\frac{1}{\left(M_{0}^{2} c^{2}\right)}\right) \cdot S_{\mu \nu} \cdot G_{v}
$$

associated with $x_{\mu}$ i.e.

$$
y_{\mu}=x_{\mu}-R_{\mu}
$$

which implies that locally extended real media in $\mathrm{I}_{0}$ are described by pairs of points as first suggested by Yukawa.

5) An inertial mass (usually not constant) $\mu_{0}$ defined by

$$
-M_{0} c^{2}=G_{\mu} \cdot \dot{x}_{\mu}
$$

can also be attributed to $x_{\mu}: M_{0}$ being located at $y_{\mu}$ since one has:

$$
\dot{y}_{\mu}=\dot{x}_{\mu}-\dot{R}_{\mu}=\dot{x}_{\mu}-\frac{1}{M_{0}^{2} c^{2}}\left(G_{\mu} \cdot \dot{x}_{v}-G_{v} \dot{x}_{\mu}\right) G_{v}=\frac{\mu_{0}}{M_{0}^{2}} \cdot G_{\mu}
$$


so that the motion of $y_{\mu}$ is locally rectlilinear and $y_{\mu}$

has a proper time $\Theta$,

(with $\quad d \lambda / d \Theta=M_{0} / \mu_{0}^{\prime}$ ) and we have :

and

$$
y_{\mu}^{\prime}=\dot{y}_{\mu} \cdot \frac{d \tau}{d \Theta}=G_{\mu} / M_{0}=\text { constant. }
$$

$$
\mu_{\mu \nu}=R_{\mu} G_{v}-R_{v} G_{\mu}+S_{\mu \nu}
$$

w.r.t. the center of gravity. Local instantaneous four rotations are described by :

- A specific « beigrössen » four-frame $b_{\mu}^{\xi}(\xi=1,2,3,0)$ with

$$
\begin{aligned}
& \dot{x}_{\mu}=b_{\mu}^{4}=\frac{i c}{6} \varepsilon_{\mu v \alpha \beta} \cdot \varepsilon^{r s t} b_{v}^{r} b_{\alpha}^{s} b_{\beta}^{t}, b_{\mu}^{\xi}=(i / 2) \varepsilon_{\mu v \alpha \beta} \dot{x}_{v} S_{\alpha \beta} \text { and } \\
& S_{\alpha \beta}=I \cdot \dot{b}_{\alpha}^{\xi} \cdot b_{\beta}^{\xi} .
\end{aligned}
$$

- A specific four-frame $a_{\mu}^{\xi}$ centered on $y_{\mu}$ with $M_{\alpha \beta}=K \cdot \dot{a}_{\alpha}^{\xi} \cdot a_{\beta}^{\xi}$ for $a_{\mu}^{4}$

along $y_{\mu}^{\prime}$ and $a_{\mu}^{3}=\left(i / 2 M_{0} c\right) \cdot \varepsilon_{\mu v \alpha \beta} G_{v} \mu_{\alpha \beta}$.

This set of relations must be completed by relations which will define the interactions between the extended elements i.e. the propagation in the aether of collective motions corresponding to observed gravitational and electromagnetic phenomena.

Before the introduction of such interactions one must recall that such proposals have already been made in the past. We only mention here:

- Weyssenhof's proposal [9] $S_{\alpha \beta} \dot{x}_{\beta}=0$ extensively discussed in the literature.

- Nakano's proposal [12] $S_{\alpha \beta} \dot{x}_{\beta}=I \cdot \ddot{x}_{\alpha}$.

- Roscoe's proposal with photon mass [13].

\section{Polarizable Vacuum Representation Of General Relativity}

Since all observed effects of gravity in distant space rest on light observation (including $\gamma$ and radio $\mathrm{em}$ waves coming through space from distant sources) a simple model endows the polarizable vacuum with properties that might account for all the phenomena in terms of distorsions. This initial proposal of Wilson and Dicke has been recently revived with astonishing success by Puthoff [5] and Krogh [14]. We first summarize their model and will complete it with a supplementary mass term in electro-magnetism.

One starts from the idea that in flat space the electric field moves in a real « vacuum medium» with a point varying dielectric constant $K$ : so that this $D$ field satisfies the vacuum equation:

$$
D=K \cdot \varepsilon_{0} \cdot E .
$$

This corresponds to a variable fine structure constant 


$$
\alpha=\frac{e^{2}}{4 \pi \varepsilon_{0} \hbar c} \cdot\left(\frac{\mu(K) / \mu_{0}}{K}\right)^{1 / 2}:
$$

so that the vacuum has permittivity and permeability constants given by

$$
\varepsilon_{0} \rightarrow \varepsilon=K \cdot \varepsilon_{0} \text { and } \mu_{0} \rightarrow \mu=K \cdot \mu_{0}
$$

and an impedance $(\mu / \varepsilon)^{1 / 2}=\left(\mu_{0} / \varepsilon_{0}\right)^{1 / 2}$ to satisfy Eötvos-type experiments. The local velocity of light for a given frequency $v$ varies like $V_{v}=c / K$ i.e like $1 /(\mu \varepsilon)^{1 / 2}$. The corresponding principle of equivalence implies that the self energy of a system changes when $K$ changes; so that a flat-space energy $E_{0}$ in flat space changes into

and one has

$$
E=E_{0} \cdot(K)^{-1 / 2}
$$

$$
m=m_{0} \cdot K^{3 / 2} .
$$

As a consequence the condition $\mathrm{E}=\hbar \cdot \omega$ becomes

$$
\omega=\omega_{0}(K)^{-1 / 2}
$$

along with the time and length variations $\Delta t$ and $\Delta r$ given by the relations:

$$
\Delta t=\Delta t_{0}(K)^{1 / 2} \text { and } \Delta r=\Delta r_{0}(K)^{-1 / 2} .
$$

These relations are evidently equivalent to a local curvature of space. Indeed a $d x_{0}$ length rod shrinks to $d_{x}=d_{x_{0}} \cdot(K)^{-1 / 2}$ and would measure $d x_{0}$, where the rod remains rigid, is now expressed in terms of $d x$-length rod as $d x_{0}=(K)^{1 / 2} d x$.

Using the same argument for $d t$ and $d t_{0}$ we find that one can write:

$$
d S^{2}=c^{2} d t_{0}^{2}-\left(d x_{0}^{2}+d y_{0}^{2}+d z_{0}^{2}\right)
$$

which transforms into

$$
d S^{2}=\frac{1}{K} c^{2} d t^{2}-K\left(d x^{2}+d y^{2}+d z^{2}\right)
$$

i.e.

$$
d S^{2}=g_{i j} \ldots d x^{i} d x^{j}
$$

with $\quad g_{00}=1 / K, \quad g_{11}=g_{22}=g_{33}=-K \quad$ and $\quad g_{i j}=0$ for $i \neq j$.

In the case of a spherically symmetric mass distribution one writes 


$$
\left\{\begin{array}{l}
K=e^{2 G \cdot M / r c^{2}} \\
K=1+2 \frac{G \cdot M}{r c^{2}}+\frac{1}{2}\left(\frac{2 G M}{r c^{2}}\right)^{2}+\ldots
\end{array}\right.
$$

where $G$ is the gravitational constant, $M$ the mass and $r$ the distance from its origin located at the center of mass. Puthoff [5] has recently shown that this model accounts (sometimes with better precision) for all known experimental tests of General Relativity in a simple way i.e. one can describe

- The gravitational redshift given by $\omega=\omega_{0} /(K)^{1 / 2}$ (so that

$$
\left.\Delta \omega / \omega \cong\left(G M / R^{2} c^{2}\right) h \text { has a } 1 / 100 \text { precision }\right) \text {. }
$$

- The bending of light rays by the sun and stars.

- The advance of the Perihelion of Mercury.

He has also shown that one can derive the form of (22) from a general Lagrangian with a variable $K$ i.e. leaving aside vacuum interaction,

$$
\begin{aligned}
& L=-\left[\frac{m_{0} c^{2}}{K^{1 / 2}}\left(1-\left(\frac{v}{(c / K)}\right)^{2}\right)^{1 / 2}+q \cdot \phi-q \cdot \vec{A} \vec{V}\right] \delta^{3}(r-\bar{r}) \\
& -\frac{1}{2}\left(B^{2} /\left(K \cdot \mu_{0}\right)-K\left(\varepsilon_{0} E^{2}\right)\right)-\frac{\lambda}{K^{2}}\left[(\nabla K)^{2}-\frac{1}{\left(c / K^{2}\right)}\left(\frac{\partial K}{\partial t}\right)^{2}\right]
\end{aligned}
$$

in $I_{0}$.

This association of gravitational theory with electromagnetic theory based on the introduction of a variable dielectric «vacuum » constant $K$ has recently been made more explicit by Krogh [14]. Noting that:

a) Electromagnetic theory implies the effects of electromagnetic vector fourpotential vectors $A_{\mu}$ on the phases $S$ of quantum mechanical waves so that one has

$$
\Delta S=\frac{q}{h} \int \phi d t-\frac{q}{h c} \int \vec{A} \cdot d \vec{S}
$$

for charged particles moving under the influence of the four vector, $A_{\mu}$.

b) If $m_{\gamma} \neq 0$ ( $m_{\gamma}$ is the mass term introduced into Maxwell's equation) the force on charged particles takes the form

$$
F=q\left(E+\frac{V \times B}{c}\right)+q \cdot V
$$

where the first term is the usual transverse Poynting force on currents and the second a longitudinal force along currents (resulting from non zero photon mass) recently observed by Graneau [11] and Saumont [16]. 
c) One can describe gravity with a four-vector density $A_{\mu}^{g}$ so that the gravitational (Newton) and electromagnetic (Coulomb) potentials have the same form, but different coupling constants. This suggests that both wave fields and singularities are just different aspects of the same fundamental field.

\section{Extension of Maxwell's Equations}

This discussion opens the possibility to test new types of extensions of Maxwell's equations in the laboratory. Since this has already been attempted some results (derived within the frame of the model) are given here:

a) From a non-zero vacuum conductivity coefficient $\sigma \neq 0[6]$ we have in vacuum $\operatorname{div} E=0$ with curl $H=\sigma E+\varepsilon_{0} \chi_{0} \partial E / \partial t$ and $\operatorname{div} H=0$ with curl $E$ $=-\mu_{0} \chi_{m} \partial H / \partial t$.

b) From an associated non-zero photon mass term $\left(m_{\gamma} \neq 0\right)$ (with $A_{\mu} A_{\mu} \rightarrow 0$ F.A.P.P.) where $A_{\mu}$ denotes the total four-potential density in Dirac's aether model. This introduces a non-zero fourth component of the current $J_{\mu}=\sigma E, j_{0}$ (where $j_{0} \neq 0$ ) into the vacuum corresponding to a real detectable space. Within the present technology this implies that the present $<<$ vacuum $>>$ really carries space-charge currents [17] (so that the divergence of the electric field is different from zero $<<$ in Vacuo $>>$ ) and the corresponding existence of a displacement current (i.e. a curl of the magnetic field) and its associated current density ${ }^{4}$.

\subsection{Massive Photons}

A unification of massive spin 1 photons piloted by electromagnetic waves built with massive extended sub-elements has been developed in a series of books by Evans, Vigier et al. [6] The model implies the introduction of spin and mass with an associated energyless magnetic field component $B^{(3)}$ in the direction of propagation and a small electrical conductivity in the Dirac vacuum also implying a new $<<$ tired light $\gg>$ mechanism $[6,22]$. Corresponding equations will be given below.

In the "absolute » inertial frame $I_{0}$ all massive particles are governed by a

gravitational potential four-vector $\phi_{g}, \vec{A}_{g} / c$, associated with a small mass $m_{g}$ which can be decomposed into transverse, longitudinal and gradiant potentials.

We can thus associate the relations

$$
\square=-\frac{\rho}{\varepsilon_{0}}+\mu \phi \quad \text { and } \square \vec{A}=-\vec{d}_{0} / \varepsilon_{0} c+\mu \vec{A}
$$

\footnotetext{
${ }^{4}$ Such attempts have been recently published in a book by Lehnert \& Roy [18] so we shall only present a summary of some results and assumptions.
} 
which represent the electromagnetic field in vacuum in any inertial frame $\Sigma_{0}$ the relations:

$$
\square \phi_{g}=4 \pi G m \rho_{\mu}+\mu_{g} \cdot \phi_{g} \text { and } \square \vec{A}_{g}=4 \pi \cdot G \cdot \vec{j}_{m}+\mu_{g} \vec{A}_{g} \text {, }
$$

which represent the gravitational field in the same vacuum; where $\rho_{\mu}$ refers to the mass density, $j_{m}$ to the mass current and $\mu$ and $\mu_{g}$ to electromagnetic and gravitational mass (both very small $\cong 10^{-65}$ grams) and $\rho \cdot c_{0}$ in the $\square$ terms

( $\left.\square=\nabla^{2}-\left(1 / c_{0}^{2}\right) \partial / \partial t^{2}\right)$ represents the corresponding wave velocities (which except in $I_{0}$ depend on the directions in flat space-time) so that one has:

$$
c_{0}=c \cdot e^{2 \phi_{g} / c^{2}}
$$

where $c$ is the value in the absence of a gravitational potential $A_{\mu}^{g}$. In this model, one assumes, with Sakharov, that the gravitational field corresponds to local depressions in the immensely positive energy of the zero-point field; and gravitational fields represent regions of diminished energy (i.e. that their momentum gravity corresponds to « holes » in vacuum energy or local defects of vacuum elements). Their effective momentum is thus opposite and corresponding gravitational forces are attractive.

Such an association also suggests that although measuring devices (i.e. observations) in local inertial Poincaré frames are altered by gravitational potentials (they are part of the same real physical background in this model). There is no effect on the geometry of flat space and time. For any given real inertial local Poincaré frame $\Sigma_{0}$ real space is Euclidean and one uses Poincaré transformations between $\Sigma_{0}$ and $I_{0}$ to describe real motions which include consequences of gravitational potentials. For example a reduction of the velocity of quantum mechanical waves, including light, is taken as a fundamental effect of gravitational potentials. Clocks are slowed and measuring rods shrink in such potentials by a factor $e^{\phi_{g} / c^{2}}$.

\subsection{Divergence of the Electromagmetic Field}

A non-vanishing divergence of the electric field given below, can be added to Maxwell's equations which results in space-charge distribution. A current density arises in vacuo and longitudinal electric non-transverse electromagnetic terms (i.e. magnetic field components) appears (like $B^{(3)}$ ) in the direction of propagation.

Both sets of assumptions were anticipated by de Broglie and Dirac. They imply that the real zero-point (vacuum) electromagnetic distribution 
- is not completely defined by $F_{\mu \nu}$ but by a four-vector field distribution given by a four-vector density $A_{\mu}$ associated with a de Broglie-Proca equation i.e.

$$
A_{\mu}\left(x_{\alpha}\right)=-\frac{m_{\gamma}^{2} c^{2}}{\hbar^{2}} A_{\mu}\left(x_{\alpha}\right)
$$

and its complex conjugated equation.

- that the $A_{\mu}$ field potential equation also contains a gradient term so that one has in vacuum (20):

$$
A_{\mu}=A_{\mu}^{T}+A_{\mu}^{L}+\lambda \partial_{\mu} S
$$

with $A_{\mu} A^{*} \rightarrow 0$ (F.A.P.P) and a small electrical conductivity in vacuo.

\section{New Possible Consequences}

Since such models evidently imply new testable properties of electromagnetic and gravitational phenomena we shall conclude this work with a brief discussion of the points where it differs from the usual interpretations and implies new possible experimental tests.

If one considers gravitational and electromagnetic phenomena as reflecting different behaviours of the same real physical field i.e. as different collective behaviour, propagating within a real medium (the «aether ») one must start with a description of some of its properties.

We thus assume

A) that this « aether » is built (i.e. describable) by a chaotic distribution

$\rho\left(x_{\mu}\right)$ of small extended structures represented by four-vectors $A_{\mu}\left(x_{\alpha}\right)$ round each absolute point in $I_{0}$. This implies

- the existence of a basic local high density of extended sub-elements in vacuum

- the existence of small density variations $\delta \rho\left(x_{\mu}\right) A_{\alpha}(x \mu)$ above $\delta \rho>0$ for light and below $(\delta \rho<0)$ for gravity density at $x_{\mu}$.

- the possibility to propagate such field variations within the vacuum as first suggested by Dirac [17].

One can have internal variations: i.e. motions within these sub-elements characterized by internal motions associated with the internal behaviour of average points (i.e. internal center of mass, centers of charge, internal rotations : and external motions associated with the stochastic behaviour, within the « aether », of individual sub-elements. As well known the latter can be analyzed at each point in terms of average drift and osmotic motions and $A_{\mu}$ distribution. It implies the indtroduction of non-linear terms. Tysis has been developed by MacGregor [4], Guerra and Pusterla and Smolin.

To describe individual non-dispersive sub-elements within $I_{0}$, where the scalar density is locally constant and the average $A_{\mu}$ equal to zero, one introduces at its central point $Y_{\mu}(\theta)$ a space-like radial four-vector $A_{\mu}=r_{\mu} \exp (i S / \hbar)\left(\right.$ with $r_{\mu} r^{\mu}=\mathrm{a}^{2}$ 
$=$ constant $)$ which rotates around $Y_{\mu}$ with a frequency $v=m_{\gamma} c^{2} / h$. At both extremities of a diameter we shall locate two opposite electric charges $e^{+}$and $e^{-}$(so that the subelement behaves like a dipole). The opposite charges attract and rotate around $Y_{\mu}$ with a velocity $\cong c$. The $+\mathrm{e}$ and $-\mathrm{e}$ electromagnetic pointlike charges correspond to opposite rotations (i.e $\pm \hbar / 2$ ) and $A_{\mu}$ rotates around an axis perpendicular to $A_{\mu}$ located at $Y_{\mu}$, and parallel to the individual sub-element's four momentum $\partial_{\mu} S$.

If one assumes electric charge distributions correspond to $\delta m>0$ and gravitation to $\delta m<0$ one can describe F.A.P.P. such sub-elements as holes $(\delta m<0)$ around a point 0 around which rotate two point-like charges rotating in opposite directions as shown in Figure 1 below.

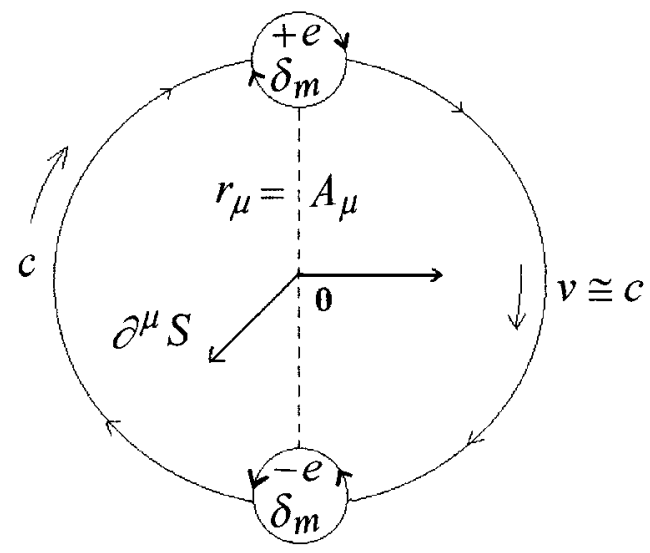

Figure 1. Conceptual diagram of two oppositely charged subelements rotating at $\mathrm{v} \cong c$ around a central point 0 behaving like a dipole « bump » and « hole » in the topology of the Dirac vacuum.

These charges themselves rotate with a velocity $c$ at a distance $r_{\mu}=A_{\mu}$ (with $r_{\mu} r_{\mu}$

$=$ Const.). From 0 one can describe this by the equation

$$
\square A_{\mu}-\frac{m_{\gamma}^{2} c^{2}}{\hbar^{2}} \cdot A_{\mu}=\frac{\left[[]\left(\mathrm{A}_{\alpha}^{*} \mathrm{~A}_{\alpha}\right)\right]^{1 / 2}}{\left(A_{\alpha}^{*} A_{\alpha}\right)^{1 / 2}} \cdot A_{\mu}
$$

with $A_{\mu}=r_{\mu} \cdot \exp \left[i S\left(x_{\alpha}\right) / \hbar\right]$ along with the orbit equations for $\mathrm{e}^{+}$and $e^{-}$we get the force equation

$$
m \cdot \omega^{2} \cdot r=e^{2} / 4 \pi r^{2}
$$


and the angular momentum equation:

$$
m_{\gamma} \cdot r^{2} \cdot \omega=\hbar / 2
$$

Eliminating the mass term between (31) and (33) this yields

$$
\hbar \omega=e^{2} / 2 r
$$

where $\mathrm{e}^{2} / 2 \mathrm{r}$ is the electrostatic energy of the rotating pair. We then introduce a solitontype solution

$$
A_{\mu}^{0}=\frac{\sin \cdot K \cdot r}{K \cdot r} \cdot \exp \left[i\left(\cot -K_{0} x\right)\right]
$$

where

$$
K=m c / \hbar, \omega=m c^{2} / \hbar \text { and } K_{0}=m v / \hbar
$$

satisfies the relation (31) with $r=\left((x-v t)^{2} \cdot\left(1-v^{2} / c^{2}\right)^{-1}+y^{2}+z^{2}\right)^{1 / 2}$ i.e.

$$
A_{\mu}^{0}=0 \text { : }
$$

so that one can add to $A_{\mu}^{0}$ a linear wave $A_{\mu} \quad$ (satisfying

$\left.A_{\mu}=\left(m_{\gamma}^{2} c^{2} / \hbar^{2}\right) A_{\mu}\right)$ which describes the new average paths of the extended wave elements and piloted solitons.

Within this model the question of the interactions of a moving body (considered as excess or defect of field density, above or below the "aether's " neighbouring average density) with a real « aether » appears immediately 5 .

As well known, as time went by, observations established the existence of unexplained behaviour of light and some new astronomical phenomena which led to discovery of the Theory of Relativity.

In this work we shall follow a different line of interpretation and assume that if one considers particles, and fields, as perturbations within a real medium filling flat space time, then the observed deviations of Newton's law reflect the interactions of the associated perturbations (i.e. observed particles and fields) with the perturbed average background medium in flat space-time. In other terms we shall present the argument (already presented by Ghosh et al. [19]) that the small deviations of Newton's laws reflect all known consequences of General Relativity

The result from real causal interactions between the perturbed local background «aether» and its apparently independent moving collective perturbations imply

5 As remarked by Newton himself massive bodies move in the vacuum, with constant directional velocities, i.e. no directional acdeleration, without any apparent relative «friction » or « drag » term. This is not the case for accelerated forces (the equality of inertial and gravitational masses being a mystery) and apparent absolute motions were proposed by Newton and later contested by Mach. 
absolute total local momentum and angular momentum conservation resulting from the preceding description of vacuum elements as extended rigid structures.

\section{Inertia And Vacuum Drag As Possible Extension of Newton's Model}

If one starts from an «aether» built with moving small extended structures with an average real distribution isotropic in an inertial frame $I_{0}$ i.e. examine the effects in a given inertial frame $I$ centered on a point $Y_{\mu}$ of the real vacuum distribution on a test particle moving with absolute velocity $V^{0}$ and angular momentum $\omega_{\alpha \beta}^{0}$ one can evaluate more precisely, the collective interactions carried by this «aether between two extended neighbouring regions centered on points $A$ and $B$ with two centers of mass situated at $\mathrm{X}_{\mathrm{A}}$ and $\mathrm{X}_{\mathrm{B}}$.

If we start with $\delta \rho<0$ i.e. gravitational effects, it appears immediately

a) that if one assumes the gravitational potential is spherical in the rest frame $I_{B}$ of its source $B$,

b) that the motion of $A$ undergoes a velocity dependent inertial induction w.r.t. $A$ i.e. a friction depending on the velocity $v$ of $A$ w.r.t. $B$

c) that this motion is also submitted to an acceleration dependent inertial w.r.t. $I_{B}$ i.e. also an acceleration depending on its acceleration a measured in $I_{B}$.

d) possible terms depending on higher order time derivations which we will neglect in the present analysis we can write (19) the force on $A$ due to $B$ in $I_{B}$ in the form $F=F_{S}+F_{v}+F_{a}$ where

$$
F=-G \cdot \frac{m_{A} \cdot m_{B}}{r^{2}}-G^{\prime} \frac{m_{A} \cdot m_{B}}{c^{2} r^{2}} \cdot v^{2} \cdot f(\theta) \hat{U}_{r}-G^{\prime \prime} \cdot \frac{m_{A} \cdot m_{B}}{c^{2} r} \cdot a f(\phi) \hat{U}_{r}
$$

The terms $G, G^{\prime}, G^{\prime \prime}$ are scalars possibly dependent on $v$. The terms $m_{A}$ and $m_{B}$ are the gravitational masses in $I_{B}, \hat{U}$, is the unit vector along $r$. $f(\theta)$ and $f(\phi)$ must have the same form i.e. $1 / 2 \cos \phi$ or $\cos \phi|\cos \phi|$. If we also accept the preceding velocity dependent analysis for contracting rods and retarded clocks then we should write $G=G^{\prime}$ in (38) and take $\mathrm{f}(\theta)==\cos \theta|\cos \phi|$ as done by Ghosh [19]. Moreover, if we compare the form given by Weber to the repulsion of two electric charges of the same sign :

$$
F_{A B}^{e}=\frac{e_{A} \cdot e_{B}}{4 \pi \varepsilon r^{2}} \cdot\left[1-\frac{1}{c^{2}}\left(\frac{d r}{d t}\right)^{2}+\frac{2 r}{c^{2}} \cdot \frac{d^{2} r}{d t^{2}}\right]
$$

corresponding to electromagnetism, with the recent form given by Assis [18] to attracting interacting masses $m_{A}$ and $m_{B}$ i.e. 


$$
F_{A B}^{g}=-G \cdot \frac{m_{A} \cdot m_{B}}{r^{2}}\left[1+\frac{6}{c^{2}}\left[r \cdot \frac{d^{2} r}{d t^{2}}-\frac{1}{2}\left(\frac{d r}{d t}\right)^{2}\right]\right]
$$

We see they have exactly the same form; the difference of their coefficients being compatible (within our interpretation) since they correspond to opposite variations of the average vacuum density. Their interpretation in terms of $\delta \rho>0$ (for electromagnetism) and $\delta \rho<0$ (for gravitation) also explains (at last qualitatively) why extended depressions repel or attract when they rotate through parallel or antiparallel directions and only attract when $\delta \rho<0$. This also explains why a reduction of attraction between two masses has been observed when one puts another mass between them (the LAGEOS satelite). In this model this similarity is indeed comparable to similar behaviours of vortices for gravitation and Tsunamis for electromagnetism on an ocean surface.

If one assumes the absolute local conservation of four-momentum and angular momentum in regions containing the preceding "aether» carrying its associated collective electromagnetic and gravitational motions one can evaluate the effects of their interactions. With a real physical «aether » there is no such thing as « free » electromagnetic or gravitational phenomena. Drag theories (described as « inertial induction ») are always present and responsible for Casimir type effects in the microscopic domain. Real consequence of the « aether» appear, at various levels, in the macroscopic and cosmological domains... as has already been suggested in the literature and tested in laboratory or astronomical phenomena. We only mention here:

1) The possible consequences of modifications of the Newton and Coulomb forces testable in the laboratory.

2) The redshift and variable velocity of electromagnetic waves which results from the rotational inertial drag of extended photons moving in vacuum: an effect already observed in light traversing around the earth [20].

3) The possible measurable existence of the redshift of transverse gravitational waves... possible in the near future.

4) Observational redshift variations of light emited by Pioneer close to the solar limb i.e. also of photons grazing a massive object [20].

5) The observed anisotropy of the Hubble constant in various directions in the sky [20] associated with various galactic densities.

6) Observed torques on rotating spheres in the vicinity of large massive bodies. This also appears in some experiments, i.e.:

a) Secular retardation of the earth's rotation.

b) Earth-moon rotation in the solar system etc.

7) Apparent evolution with time of angular momentum in the solar-planetary system.

8) Different variation of redshift of light travelling up and down in the Earth's gravitational field... Which also supports existence of photon mass. 


\section{Conclusions}

As stated in the introduction of this paper, this model exploits :

a) the analogy (underlined by Puthoff) between the four vector density representation of gravity and electromagnetism in flat space-time [5]

b) the possibility of describing the causality of quantum mechanical phenomena in terms of extended solitons piloted i.e. by quantum mechanical potentials, by real guiding collective waves on a chaotic, polarizable Dirac-type aether - both moving in a flat space-time [20].

c) the representation of this «real vacuum» (Dirac aether) in terms of the chaotic distribution of real extended elements moving in the flat space-time.

d) the introduction of internal motions within extended sub-elements and their relation with local collective motions i.e. the $E=m c^{2}=h v$ relation

e) the representation of the electron (and its associated pilot-wave) in terms of extended elements with a point-like charge rotating around a center of mass [20].

These assumptions yield realistic physical characteristics to known empirical properties and predict new testable relations besides known properties of elementary particles. The present model must thus be extended, by associating new internal motions to these known properties and interpret them in terms of new strong spin-spin and spin-orbit interactions. The new predictions should be confronted experimentally to see if they represent a valid starting point for further theoretical and experimental developments.

One of the justifications of the present attemp is the existence of electromagnetic phenomena not explained by Maxwell's equations. As discussed by Barrett [21] Maxwell's theory does not explain the Aharonov-Bohm (AB) effect and Altahuler-Aharonov-Spivak (AAS) effects. It does not cover the topological phase question i.e. the Berry-Aharonov-Anandan, Pancharatnam and Chio-Wu phaserotation effects. An inclusion of Stoke's theorem is necessary and results of Ehrenberg and Siday must be analysed. The quantum results of Josephson, Hall, de Haas and van Alphen Sagnac-type experiments also need clarification. In other words Maxwell and his direct followers have discovered a continent. Its exploration of still lies in front of us !

\section{Appendix}

The unification of gravity with electromagnetism attempted by Sakharov and Zeldovich rests on the following ideas :

a) Gravitation results from perturbations of the zero point field (Z.P.F.)

b) It results from a radiation pressure of the zero-point field: i.e. $\delta \rho<0$ in this paper,

- Sakharov has proposed to describe $G_{c}$ (Newton-Covendish constant) by

$$
G_{c}=\frac{\lambda_{\rho}^{2} c^{3}}{\hbar}
$$


where $G$ is the gravity vector, $\lambda_{\rho}=\left(\hbar G_{c} / c^{3}\right)^{1 / 2}$ is the Planck length and

$$
G_{c}=e^{2} / m_{c} m_{\rho} \cdot \alpha \exp \left(-2\left[\frac{m_{p}}{m_{e}}\right]\right)^{1 / 2}=6.668
$$

- Gravity thus corresponds to variations of the $\vec{E} \times \vec{B}$ drift field

$$
G=\frac{\dot{E} \times B_{0} c}{B_{0}^{2}}=\frac{\dot{S}}{2 U_{0}} \text { with } U_{0}=\frac{B_{0}^{2}}{8 \pi}
$$

Where $S=$ Poynting vector $(E \times B \cdot c) / 4 \pi$.

c) A perturbed Z.P.F. modifies gravity.

\section{References}

1. A. Einstein, Geometry and Experience Sidelights on Relativity, Denver, 1922

2. L. Bass and E. Schrödinger, Proc.Roy.Soc.A 232, 1955

3. J.C. Maxwell, A Treatise on Electricity and Magnetism, Dover (1954).

4. H. Mac Gregor, Stationary Vacuum Polarization, (2000).

5. H.E. Puthoff, Polarizable-Vacuum, Representation of General Relativity, 1999, Austin and Phys.Rev.A, 1989

T.B. Andrews, Observed Tests and Theory of the Static Universe, 2000, preprint.

6. M.W. Evans and J.P. Vigier, The Enigmatic Photon, Kluwer, 1996

7. L. de Broglie, Mécanique Ondulatoire du Photon, Paris: Gauthier-Villas (1955).

L. de Broglie and J.P. Vigier, Phys. Rev. Let.

D. Bohm \& J-P Vigier, Phys. Rev. 109 (1958), p. 1882

C. Levitt et al. Unpublished.

8. H. Arp, Quasar Redshift and Controversies Interstellar Media, USA, 1987

9. J.V. Weyssenhof and A. Raabi, Act.Phys.Pol. 9, Kluwer, Ed.

10. J.P. Vigier, Phys.Lett.A., 235, 1997

11. P. Graneau, 2002, Why does lightning explode and generate MHD, preprint

12. I. Nakano, Progress in Theoretical Physics, (1956), 15.

13. D.F. Roscoe, Maxwell'sequations, 1997, preprint.

14. K. Krogh, Gravitation without Curved Space -Time, 2002, Submitted to General Relativity \& Gravitation.

15. F. Halbwachs, J.M. Souriau and J.P. Vigier, Journal de Physique et de Radium, 1922, 22, p. 26. See also F. Halbwachs, Théorie Relativiste. Fluides . Paris : Gauthier-Villars, 1960.

16. F. Saumont, Proc. Cold Fusion and Now Energy Symposium, Manchester, 1998

17. P.A.M. Dirac, Nature, 1951, 906

18. B. Lehnert, \& S. Roy, Extended Electromagnetic Theory, World Scientific, 2000.

19. A. Ghosh, Origin of Inertia, Apeiron, Montreal, 2000.

20. R. Pound and G.A. Rebka, Physical Review Letters 4, 337, 1960. J-P Vigier \& S, Maric, to be published.

21. T.W. Barrett, Electromagnetic Phenomena not Explained by Maxwell's Equations: Essays on the Formal Aspects of Electromagnetic Theory. Singapore: World Scientific, (1993).

22. Amoroso, R.L., Kafatos, M. \& Ecimovic, P. 1998, The origin of cosmological redshift in spin exchange vacuum compactification and nonzero restmass photon anisotropy, in G. Hunter, S. Jeffers \& J-P Vigier (eds.), Causality \& Locality in Modern Physics, pp. 23-28, Dordrecht: Kluwer.

23. R.L. Amoroso, The Origin of CMBR as Intrinsic Blackbody Cavity-QED Resonance Inherent in the Dynamics of the Continuous State Topology of the Dirac Vacuum, in R.L. Amoroso, G. Hunter, M. Kafatos \& J-P Vigier (eds.), Gravitation and Cosmology: From the Hubble Radius to the Planck Scale, 2002 Dordrecht: Kluwer. 